\title{
gु \\ Surface diffusion constants of large organic molecules determined from their residence times under a scanning tunneling microscope tip
}

\author{
J. Ikonomov, ${ }^{1}$ P. Bach, ${ }^{1}$ R. Merkel, ${ }^{2}$ and M. Sokolowski ${ }^{1, *}$ \\ ${ }^{1}$ Institut für Physikalische und Theoretische Chemie, Universität Bonn, Wegelerstr. 12, 53115 Bonn, Germany \\ ${ }^{2}$ Institut für Bio- und Nanosysteme 4, Forschungszentrum Jülich, 52425 Jülich, Germany
}

(Received 20 March 2010; published 27 April 2010)

\begin{abstract}
We introduce a method to determine the lateral diffusion constants of planar organic molecules adsorbed on surfaces from the temporal width $\tau$ of pulses in the tunneling current measured for a stationary scanning tunneling microscope tip. The method uses the molecular footprint as a scale bar and evaluates $\tau$ as the first-passage time. Temperature-dependent diffusion constants are reported for the two model molecules copper phthalocyanine and 3,4,9,10-perylene-tetracarboxylic-dianhydride adsorbed on the $\operatorname{Ag}(100)$ surface.
\end{abstract}

DOI: 10.1103/PhysRevB.81.161412

PACS number(s): 68.43.Jk, 68.35.Fx, 82.37.Gk

Diffusion of adsorbates on a surface is a highly relevant fundamental process and has hence been of interest for a long time. ${ }^{1}$ Surface diffusion determines, e.g., the kinetics of catalytic and growth processes. In addition, the related activation energies yield insight into the fundamental adsorbatesubstrate interaction, namely, the lateral corrugation of the bonding potential, which can serve as a further test parameter for theoretical investigations in this field. ${ }^{2}$ Most directly, the diffusion coefficients are obtained by particle tracking. In earlier days, this was performed by field ion microscopy, ${ }^{3}$ and, today, by fast scanning tunneling microscopy (STM). ${ }^{4-8}$ Other, more indirect, methods are based on the temporal evolution of concentration profiles of adsorbates. ${ }^{9,10}$

Alternatively to the tracking method, information on the kinetics of particles on a surface can be obtained from the local residence time of the adsorbate on a defined site. This information can be, in particular, extracted from the time dependence of the current $I(t)$ measured under a stationary STM tip. Pulses of temporal width $\tau$ appear in the current signal, if an atom or molecule diffuses into the tunnel junction under the STM tip and resides there for a time $\tau$ until it diffuses out of the junction again. Commonly, these pulses are attributed as "noise" when STM measurements are performed on a two-dimensional (2D) gas phase of an adsorbate. In Fig. 1(a) such pulses are illustrated. As we will demonstrate here, it is possible to determine diffusion constants from these pulses which is, in particular, beneficial, if the diffusion is too fast to allow tracking.

Experiments evaluating this signal were, to our knowledge, reported only rarely. Binnig et al. ${ }^{11}$ recorded $I(t)$ spectra for $\mathrm{O} / \mathrm{Ni}(110)$ and $\mathrm{Ni}(100)$ but were limited by the speed of their electronics. Lozano and Tringides ${ }^{12}$ were, to our knowledge, the first, who successfully analyzed the fluctuations in the tunnel current and determined the activation energy of the diffusion of oxygen on the Si(111) surface. The determination of hopping rates was recently reported for $\mathrm{Au}$ atoms on $\mathrm{Si}(7 \times 7)$ by Wang et al. ${ }^{13}$ However, for the noted atomic, and hence on the lateral scale of the tunnel junction pointlike adsorbates, the diffusion constants cannot be directly obtained, since a geometric scale bar is missing in the process. This is principally different for larger planar organic molecules which exhibit a finite lateral size with respect to the STM tip, as illustrated in Fig. 1(b). Here the residence time $\tau$ will also include the diffusion steps of the molecule under the tip, as long as the molecule supports the higher tunneling current. Consequently, $\tau$ depends on the size of the molecule which hence provides an inherent scale bar and makes absolute diffusion constants accessible from the $I(t)$ spectra. As we demonstrate here, this method is also fast and can be used to measure the diffusion coefficients in specific local regions on a surface.

We have studied the diffusion of two organic prototype (a)

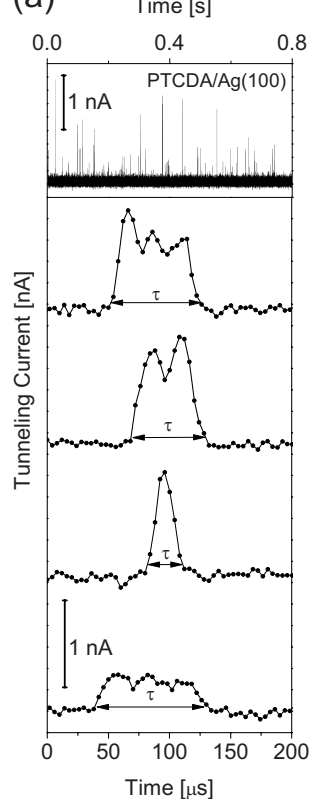

(b)

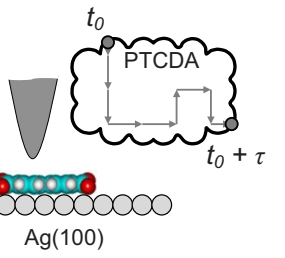

(c)

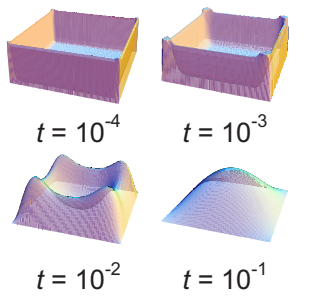

FIG. 1. (Color online) (a) Part of a current-time spectrum with magnified exemplary pulses. The residence times $\tau$ of the molecules under the tip are determined from the FWHM as indicated in the spectrum. (b). Principal schema of the measuring process (top and side view). The trajectory of the STM tip with respect to the molecule (PTCDA) is indicated. $t_{0}$ and $t_{0}+\tau$ denote the "entrance" and "exit" times. (c) Time evolution of the probability density $p(x, y)$ of the STM tip on a quadratic molecule (size $L \times L$ ). Times $t$ are in units of $L^{2} / D$; heights are scaled with a factor $\exp (2 t)$. The small peaking of $p(x, y)$ at the four corners stems from the particular chosen start density, $p(x, y, t=0)$ and is not relevant for the evaluation. 


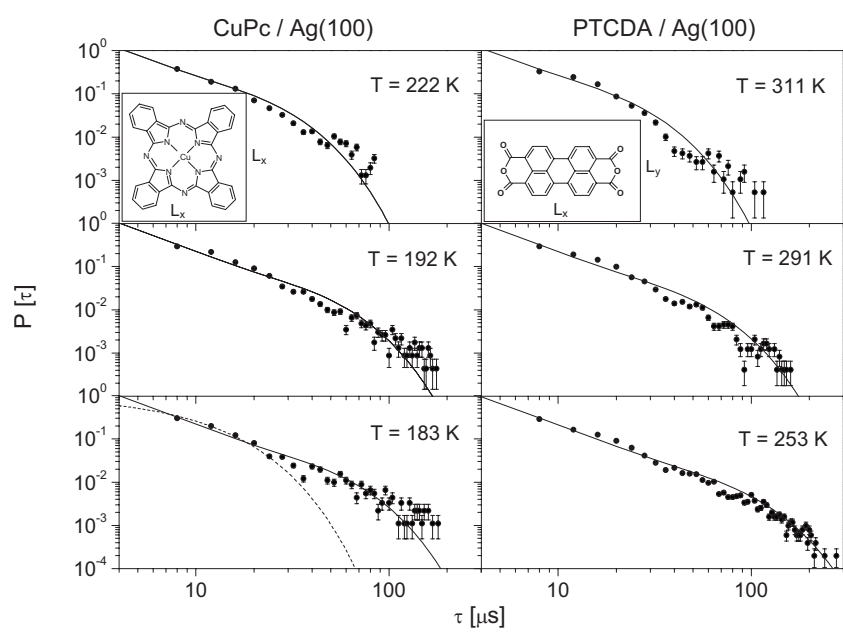

FIG. 2. Exemplary residence time spectra $P(\tau)$ measured for $\mathrm{CuPc}$ and PTCDA diffusing on $\mathrm{Ag}(100)$ at different temperatures. The full black lines are fits to the data according to the model of diffusive motion of an extended planar molecule versus the stationary STM tip, as described in the text, using isotropic diffusion constants, i.e., $D_{x}=D_{y}$. The dashed fit curve (given for $\mathrm{CuPc}$ ) shows the exponential decay curve that is expected for a pointlike object. The error bars indicate the statistical uncertainty. The insets show structure formulas of the respective molecules with indicated molecular footprints. CuPc: $L_{x}=11.9 \AA$; PTCDA: $L_{x}=14.2 \AA$ and $L_{y}=9.2 \AA$.

molecules on the $\operatorname{Ag}(100)$ surface, namely, copper phthalocyanine (CuPc) and 3,4,9,10-perylene-tetracarboxylicdianhydride (PTCDA) (formulas are given in Fig. 2, below). $\mathrm{CuPc}$ was, in particular, chosen for its high symmetry which implies an isotropic diffusion on $\operatorname{Ag}(100)$, whereas, for PTCDA, different diffusion constants in the direction of the long $\left(D_{x}\right)$ and short axes $\left(D_{y}\right)$ may be expected. For both molecules submonolayers with about $10-15 \%$ coverage were prepared by vacuum deposition. At this coverage both molecules form islands which coexist with a 2D gas phase. For both molecules the lateral arrangement in the ordered island is commensurate with the $\operatorname{Ag}(100)$ surface, which points to relevant molecule substrate interactions. ${ }^{14,15}$

The geometric footprints of CuPC and PTCDA were approximated by a square $\left(L_{x} \times L_{x}\right)$, respectively a rectangle $\left(L_{x} \times L_{y}\right)$, as indicated in Fig. 2. These dimensions were estimated from the van der Waals contours of the molecules. The footprints correspond well to the images of the planar molecules seen by STM. Under the approximation of a pointlike STM tip [as illustrated in Fig. 1(b)], a tunneling current through the molecule is supported as long as the tip is located within the footprint. Since the periodicity of the underlying $\operatorname{Ag}(100)$ surface $(a=2.89 \AA)$ is small compared with the molecular footprints, it is plausible and indicated by our results that the molecules undergo several diffusion jumps between entering and leaving the tunnel junction. These jumps under the tip can be observed as height fluctuation of $I(t)$ within individual pulses [see Fig. 1(a)]. Hence typical diffusion jumps are significantly smaller than the extension of the molecule. However, we note that the occurrence of long jumps, ${ }^{5}$ is not in conflict with our determination of the diffusion constant from the data since we use a continuum model which is independent of a specific jump length.

The variable temperature STM was equipped with mechanically cut Pt/Ir tips. ${ }^{15}$ Only tips which gave good and reproducible molecular resolution were used. For the recording of the $I(t)$ curves, the STM tip was positioned on the gas phase at preset tunnel parameters of $U_{\text {bias }}=1.7 \mathrm{~V}$ (tunneling into unoccupied molecular states) and a time averaged $I_{a v} \approx 20 \mathrm{pA}$. The sample temperatures were between 140 and $230 \mathrm{~K}$ for $\mathrm{CuPc}$, and between 235 and $310 \mathrm{~K}$ for PTCDA. The temperature difference reflects the difference in the phase diagrams and diffusion constants of the two adsorbates on $\operatorname{Ag}(100)$.

The tunnel currents were recorded for times of $0.8-8 \mathrm{~s}$ on a digitization grid of $4 \mu \mathrm{s}$. The lateral drift of the STM $\left(0.2-0.6 \AA^{-1}\right)$ can be neglected on this time scale. The bandwidth of the preamplifier was $150 \mathrm{kHz}$. Both, the digitization grid limited by the software and the band width of the preamplifier set a lower boundary for the detectable residence times. Typical $I(t)$ spectra contained about 5000-10 000 pulses. If the experiments were performed on an ordered island, no pulses were seen, which is understandable, since there are no molecules in the second layer of an island. We note that the sum of the full width at half maximum (FWHM) of all pulses in an $I(t)$ spectrum divided by the sampling time yields in good approximation the local time-averaged coverage under the STM tip. These values were below $1 \%$ of a closed monolayer. Hence the coverages were small and intermolecular interaction can be less expected. However, strictly speaking the here determined diffusion coefficients have to be addressed as "chemical" diffusions constants. ${ }^{1}$ From STM observations there were no indications for cluster diffusion. ${ }^{16}$

For the evaluation the residence times $\tau$ were determined from the $I(t)$ spectra as the FWHM of the pulses above the baseline [see Fig. 1(a)]. The probabilities $P(\tau)$ of pulses with a residence time $\tau$ were then computed on a grid of $4 \mu \mathrm{s}$. We note that the $I(t)$ spectra can be alternatively evaluated by computing the autocorrelation functions, ${ }^{12}$ as we have tested. However, this approach appeared numerically less robust and less direct concerning its interpretation. Examples of calculated residence time spectra $P(\tau)$, taken at different temperatures of both molecules, are displayed in Fig. 2. Remarkably, $\tau$ values span a range of nearly two orders of magnitude. However, for long residence times of $\tau \approx 100 \mu \mathrm{s}, P(\tau)$ drops to about $0.2 \%$. One can also clearly see the influence of the temperature $(T)$ for both molecules. The spectra recorded at lower $T$ fall of more slowly, which is understandable from the smaller diffusion constants. As a first test, we have plotted the $P(\tau)$ spectrum that one expects for an atom, i.e., a pointlike object. It is given by an exponential curve $\sim \exp (-h t)$, were $h$ denotes the hopping rate. ${ }^{5}$ Obviously, this curve does not fit to the data at all (see Fig. 2, dashed line). The discrepancy is substantial and cannot be lifted by a variation in the hopping rate. It indicates that the finite size of the molecule, i.e., its footprint, plays a decisive role here, or which is equivalent, that the average length of the elementary diffusion jumps are small with respect to the size of the molecule. Long diffusion jumps must hence occur with minor probability only. 
Before we proceed further in the analysis, we comment on interaction of the molecule with the STM tip. Obviously this would also influence the $P(\tau)$ spectra by attracting or repelling molecules into or out of the tunnel junction. We tested this explicitly by systematic variations in the bias voltage over a wide range. Indeed, we found increasing molecular attraction at small positive bias voltages and concomitant small tip to surface distances but vanishing effects for bias voltages $U_{\text {bias }}$ above $1.6 \mathrm{~V}$ and $I_{a v} \approx 20 \mathrm{pA}$. For all diffusion experiments we hence used $U_{\text {bias }}>1.6 \mathrm{~V}$ to exclude tip effects.

For the quantitative evaluation of the residence time spectra it is helpful to envisage the process from the viewpoint of the tip: the tip diffuses into the area defined by the footprint of molecule at some time $t_{0}$ and diffuses out of this area at $t_{0}+\tau$ [see Fig. 1(b)]. The corresponding probability density $p(x, y, t)$ of the tip can be described by the two-dimensional diffusion equation

$$
\partial / \partial t p(x, y, t)=D_{x} \partial^{2} / \partial x^{2} p(x, y, t)+D_{y} \partial^{2} / \partial y^{2} p(x, y, t)
$$

Hereby $x$ and $y$ are defined in the coordinate system of the molecule (see Fig. 2). This continuum model ignores the surface corrugation and the presence of preferential sites. It is nevertheless a good approximation, since, as deduced above, the elementary diffusion jumps are small with respect to the molecular size. At $t_{0}, p(x, y)$ is mainly located close the periphery of the molecule. With elapsing time, $p(x, y)$ diffuses into the center of the molecule while at the same time the integral over $p(x, y)$ diminishes since the tip also diffuses out of the molecule at its periphery. This is illustrated in Fig. 1(c). Important is the boundary condition which describes the off-diffusion of the tip from the molecule: $p(x, y)$ vanishes on the periphery of the molecule for all times. In general, this situation is known as the "first passage time problem" with absorbing boundary conditions. It possess an analytical solution for a rectangular area ${ }^{17}$ which we used.

From $p(x, y, t)$ the residence time spectra $P(\tau)$ can be obtained straightaway as: $P(\tau)=-\left.\frac{d}{d t}\right|_{\tau} \int_{\Omega} p(x, y, t) d x d y$, whereby the integral extends over the area $\Omega$ of the molecule. Important is the diffusion of $p(x, y)$ to the center of the molecule. This causes the observed nonexponential decay of $P(\tau)$. In Fig. 2, the respective fits to the experimental residence time spectra are shown. ${ }^{18}$ The curves were computed on the basis of identical diffusion constants $D_{x}$ and $D_{y}$ along both principal axes of the molecules $\left(D=D_{x}=D_{y}\right)$. In order to correctly fit the small values of $P(\tau)$ at large values of $\tau$, the fitted curves were computed by minimizing $\sum_{i}\left[\ln \left[P_{\text {exp }}\left(\tau_{\mathrm{i}}\right)\right]-\ln \left[P_{\text {fit }}\left(\tau_{\mathrm{i}}\right)\right]\right\}^{2}$. As a result, all data are fitted over several decades in $P(\tau)$ and $\tau$ with high accuracy. The good agreement demonstrates the applicability of our continuum model to the situation. Rotational diffusion around the center of mass of the molecules was so far not considered in the evaluation. However, we do not expect significant corrections. In the case of $\mathrm{CuPc}$, rotations should have a minor effect on $I(t)$ because of the quadratic footprint of the molecule. In the case of PTCDA, rotational diffusion is less likely due to its stronger interaction with the $\operatorname{Ag}(100)$ surface. ${ }^{17}$

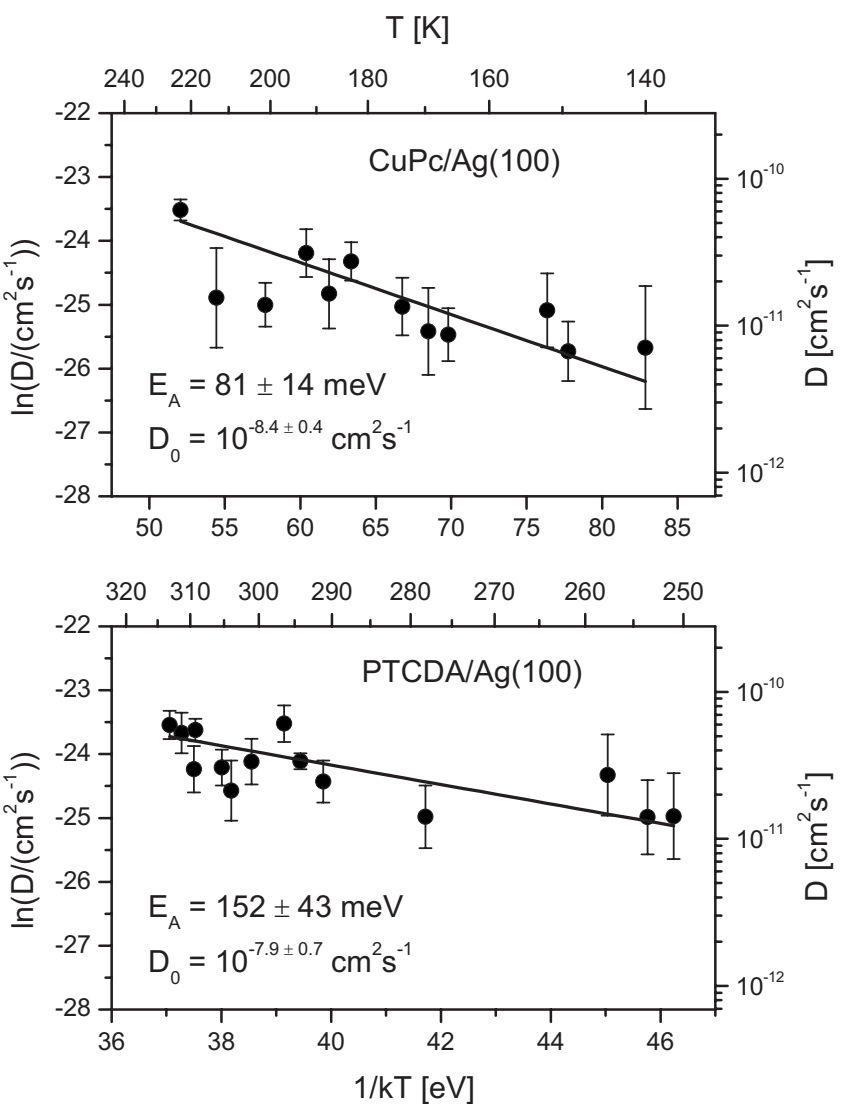

FIG. 3. Arrhenius plots of the diffusion constants with corresponding fits for $\mathrm{CuPc}$ and PTCDA on $\mathrm{Ag}(100)$. The error bars indicate the accuracy of the respective fits of the $P(\tau)$ spectra and were accounted in the fitting. Further details see text.

Arrhenius plots of the diffusion constants measured at different temperatures are displayed in Fig. 3. From least square fits $\left[D=D_{0} \exp \left(-E_{A} / k_{B} T\right)\right]$ we find the respective activation energies to be $E_{\mathrm{A}}^{\mathrm{CuPC}}=81 \pm 14 \mathrm{meV}$ and $E_{\mathrm{A}}^{\mathrm{PTCDA}}$ $=152 \pm 43 \mathrm{meV}$. Obviously the activation energy for CuPc is about a factor of about two smaller than that for PTCDA. Intuitively, one relates this difference to a stronger corrugation of the surface potential for PTCDA compared to $\mathrm{CuPc}$ related to a stronger bonding. From the fitted parameters in Fig. 3, room-temperature values of the respective diffusion constant are calculated to be $D^{\mathrm{CuPc}}=1.7 \times 10^{-10} \mathrm{~cm}^{2} \mathrm{~s}^{-1}$ and $D^{\mathrm{PTCDA}}=4.0 \times 10^{-11} \mathrm{~cm}^{2} \mathrm{~s}^{-1}$.

For PTCDA we have also considered the possibility of different diffusion constants $D_{x}$ and $D_{y}$ along the two principle axes of the molecule. Anisotropic diffusion is conceivable for PTCDA, since, for instance, the collective movement of the two carboxylic $\mathrm{O}$ atoms of the terminal anhydride groups in the direction along or perpendicular to the long molecular can be related to different activation energies. However, performing fits with independent values of $D_{x}$ and $D_{y}$ (not shown) yielded spectra that varied from those performed with $D_{x}=D_{y}$ only very marginally, in particular, within the scattering of the experimental data points. As a consequence, we conclude that anisotropy in the diffusion constants has to be excluded or is too small to be discriminated from the present data. 
Quantitative values of diffusion constants of organic molecules on surfaces are very rare so far and show large variation, ${ }^{1}$ which limits the comparison. For example, for the planar decacyclene (DC) molecule on $\mathrm{Cu}(110)$ Schunack et al. ${ }^{5}$ obtained a value of $E_{A}=710 \mathrm{meV}$ by tracking. Notably, this molecule exhibits a diffusion constants which is about two orders of magnitude smaller $\left(D^{\mathrm{DC}}=1.2 \times 10^{-13} \mathrm{~cm}^{2} \mathrm{~s}^{-1}\right)$ at room temperature compared to those of CuPc and PTCDA on $\mathrm{Ag}(100)$. A likely reason for this is that the bonding potential for $\mathrm{DC}$ on $\mathrm{Cu}(110)$ exhibits a much higher corrugation with respect to that of $\mathrm{CuPc}$ and PTCDA on $\operatorname{Ag}(100)$ due to the more open structure and higher density of electronic states near the Fermi edge of the $\mathrm{Cu}(110)$ surface with respect to the $\mathrm{Ag}(100)$ surface. $^{19}$

As demonstrated, the above-described method allows a quantitative, fast, straightforward and local determination of the diffusion constants of planar molecules on surfaces. An obvious limitation to the precision is the accuracy by which the molecular footprint which supports the tunneling current can described. The method exploits that the molecule performs several diffusion jumps when it undergoes a displacement of its extensions, i.e., $L_{x}$ and $L_{y}$. We expect this to be the case for $\pi$ conjugated molecules which chemical interact with the surface and tend to form commensurate structures. The above criterion will be likely fulfilled in this case since the surface corrugation naturally determines the elemental jump length of diffusion. Concerning the time resolution $\Delta \tau_{\exp }$ of the STM, the method will be applicable as long as $L_{x}^{2} / D_{x} \gg \Delta \tau_{\exp }$ and $L_{y}^{2} / D_{y} \gg \Delta \tau_{\text {exp }}$. The strongest aspect of the introduced method is that it can be applied when the diffusion is too fast to allow tracking methods, as it is the case for many $\pi$ conjugated molecules on surfaces. In addition it opens the possibility to measure diffusion on small defined local surface areas, e.g., between steps, or local islands.

We thank Ch. Schmitz for experimental help and E. Barkai for theoretical advice. Support by the DFG is acknowledged.

\footnotetext{
*Corresponding author. FAX: +49 (0)228-73 2551;

sokolowski@pc.uni-bonn.de

${ }^{1}$ J. V. Barth, Surf. Sci. Rep. 40, 75 (2000).

${ }^{2}$ M. Rohlfing and T. Bredow, Phys. Rev. Lett. 101, 266106 (2008).

${ }^{3}$ G. L. Kellogg, Surf. Sci. Rep. 21, 1 (1994).

${ }^{4}$ G. Briner, M. Doering, H.-P. Rust, and A. M. Bradshaw, Science 278, 257 (1997).

${ }^{5}$ M. Schunack, T. R. Linderoth, F. Rosei, E. Lægsgaard, I. Stensgaard, and F. Besenbacher, Phys. Rev. Lett. 88, 156102 (2002).

${ }^{6}$ J. Weckesser, J. V. Barth, and K. Kern, Phys. Rev. B 64, 161403(R) (2001).

${ }^{7}$ T. Tansel and O. M. Magnussen, Phys. Rev. Lett. 96, 026101 (2006).

${ }^{8}$ L. J. Lauhon and W. Ho, J. Chem. Phys. 111, 5633 (1999).

${ }^{9}$ G. A. Reider, U. Höfer, and T. F. Heinz, Phys. Rev. Lett. 66, 1994 (1991).

${ }^{10}$ J. L. Brand, M. V. Arena, A. A. Deckert, and S. M. George, J. Chem. Phys. 92, 5136 (1990).
}

${ }^{11}$ G. Binnig, H. Fuchs, and E. Stoll, Surf. Sci. Lett. 169, L295 (1986).

${ }^{12}$ M. L. Lozano and M. C. Tringides, Europhys. Lett. 30, 537 (1995).

${ }^{13}$ K. Wang, C. Zhang, M. M. T. Loy, and X. Xiao, Phys. Rev. Lett. 94, 036103 (2005).

${ }^{14}$ P. Bach, Diploma thesis, University of Bonn, 2009.

${ }^{15}$ J. Ikonomov, O. Bauer, and M. Sokolowski, Surf. Sci. 602, 2061 (2008).

${ }^{16}$ G. L. Kellogg and A. F. Voter, Phys. Rev. Lett. 67, 622 (1991).

${ }^{17}$ S. Redner, A Guide to First-Passage Processes (Cambridge University Press, Cambridge, 2001), p. 41.

${ }^{18}$ The data point at $t=4 \mu$ s was systematically excluded in order to avoid possible artefacts due to the limit in the experimental time resolution.

${ }^{19}$ For a comparison of bonding on the noble metal surfaces see, e.g., A. Hauschild, R. Temirov, S. Soubatch, O. Bauer, A. Schöll, B. C. C. Cowie, T.-L. Lee, F. S. Tautz, and M. Sokolowski, Phys. Rev. B 81, 125432 (2010). 